\title{
Study on Vibration Characteristics of Gun Barrel Under the Action of Moving Projectile
}

\author{
Yang Zhao 1, a, Qizheng Zhou ${ }^{2, b}$ and Pengfei Yue ${ }^{3, c}$ \\ Department of Weaponry Engineering,Naval University of Engineering,Hubei Wuhan 430033,China \\ a1573380179@qq.com, bzqizheng@126.com, ‘ypenfei@163.com
}

\begin{abstract}
Keywords:gun;barrel vibration;gap between projectile and barrel;projectile-barrel coupling; numerical simulation

Abstract: This paper studied the vibration characteristics of barrel excited by projectile in lauch process of gun. Considering the effect of inertia and the contact condition between projectile and barrel, we simplified the barrel as the Euler equal cross section, and established the vibration equation of barrel which was excited by accelerated projectile, and used the modal analysis method to solve the equation. Through the simulation, we studied the influence of gap between projectile and barrel on the barrel vibration with different motion parameters, eccentric mass and continuous firing of projectile. The results showed that controlling the acceleration of projectile in chamber reasonable, reducing the gap between projectile and barrel and the eccentric mass of projectile, will help to reduce the vibration of muzzle caused by artillery launch. The research results can provide reference for the overall design of gun.
\end{abstract}

\section{Introduction}

During the firing process, the vibration of barrel is one of the main causes of the initial disturbance of projectile, which affects the firing accuracy of gun seriously. The barrel vibration is influenced by many factors, such as machining defects and weight curve of barrel, Bourdon effect, gravity and eccentric force of moving projectile, and the rotating friction torque in the chamber. The vibration of barrel is coupled with the motion of projectile under the motion of high-speed projectile. The research of the Projectile-barrel Coupling has experienced from the research on single barrel or projectile to the research on interaction of projectile and gun. Shi Yuedong ${ }^{[1,2]}$ deduced the analytical solution of the series of barrel vibration equation by using small parameter method. And the numerical simulation is carried out for the vibration characteristics of barrel with different motion parameters . Liu Ning ${ }^{[3]}$ put the projectile as an elastic ball, established the contact and collision model between projectile and barrel, and analyzed the influence of the initial position of the projectile and the gap between projectile and barrel on the vibration of gun muzzle. According to Bernoulli-Euler elementary beam theory, Jiang $\mathrm{Mu}^{[4-6]}$ studied the vibration which were caused by the acceleration of projectile in barrel. In the past analysis of the oretical modeling,the contact and collision model between projectile and barrel was relatively simple. The model simplified the projectile as an elastic sphere, without considering the perturbation of projectile in motion. Both of the centering department before the projectile and the Link Belt will contact or impact the inner wall of barrel in the actual movement of projectile in barrel. The projectile will produce small angle perturbation and more complex excitation to the barrel. At the same time, the perturbation will also affect the movement of projectile, and form initial disturbance.In addition, there was less research on the vibration of barrel under the condition of eccentric mass and continuous firing of projectile.

Based on the study and the Contact model between centering department before projectile or Link Belt and the barrel,we established the coupling vibration model between projectile and barrel. We mainly analyzed the influence of the gap between projectile and barrel on the barrel under the action of moving projectile, and we further investigated the vibration response under the eccentric mass and continuous firing condition. The conclusion can provide reference for the overall design and structure optimization of gun and ammunition. 


\section{Lateral Vibration Model of Barrel With the Moving Projectile}

We simplified the barrel as the Euler equal cross section, and assumed that the movement of projectile along the axis of chamber. We had simplified the contact model of projectile and bareel for the model of two point contact with the barrel respectively ofcentering department before the projectile and Link Belt. Considering the influence of the system vibration characteristics from the gap between moving projectile and barrel, the collision model of the moving projectile and the barrel coupling system was established.The tangential collision and friction effect between projectile and barrel were not count. The mechanical model was shown in Figure1.

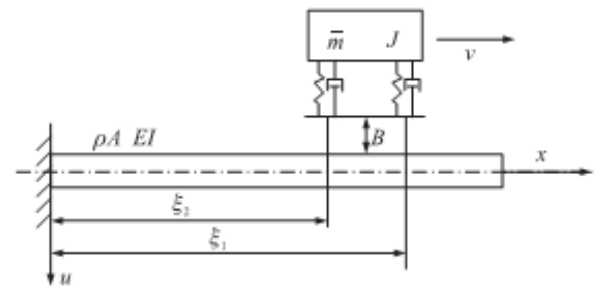

Fig.1 Collision model of projectile and barrel

Assuming that the beam length is $l$, the cross section area is $A$, the material density is $\rho$, the bending rigidity is $E I$, and the pellet quality is $m$. Then the bending vibration equation of the barrel is

$$
\left\{\begin{array}{c}
E I \frac{\partial^{4} y(x, t)}{\partial x^{4}}+2 H \rho A \frac{\partial y(x, t)}{\partial t}+\rho A \frac{\partial^{2} y(x, t)}{\partial t^{2}}=P_{1} \delta\left(\xi_{1}-x\right)+P_{2} \delta\left(\xi_{2}-x\right) \\
\xi_{1}-\xi_{2}=l_{0} \cos \theta
\end{array}\right.
$$

In the formula, $P_{1}$ and $P_{2}$ are the load of the centering department before the projectile and the Link Belt respectively. $\xi_{1}$ and $\xi_{2}$ are the displacement at the $t$ moment of the projectile respectively. $E I$ is the bending strength of barrel. $\rho$ is the mass per unit length of barrel. $A$ is the cross-sectional area of barrel. $H$ is the damping coefficient, $y(x, t)$ is the lateral displacement at the $t$ moment in $x$ site of barrel. $l_{0}$ is the distance of the action sites of centering department before projectile and Link Belt to the barrel respectively. $\delta$ is the function of Dirac, $\theta$ is the angular displacement of projectile. The differential equation of motion of the projectile is

$$
\left\{\begin{array}{c}
m\left(\frac{d^{2} s_{r}(t)}{d t^{2}}+\frac{d^{2} y(x, t)}{d t^{2}} \delta\left(\xi_{0}-x\right)\right)=\left(P_{1}+P_{2}\right)-m g \\
J \ddot{\theta}=P_{1} l_{1}-P_{2} l_{2}
\end{array}\right.
$$

In the formula, $J$ is the pitch moment of inertia of projectile. $\theta$ is the angular displacement of projectile. $s_{r}(t)$ is the lateral displacement of the centroid of projectile to the axis of barrel. $\xi_{0}$ is the longitudinal displacement of the centroid of projectile to the axis of barrel. $l_{1}$ and $l_{2}$ are the distance of the action sites before and after of the projectile to the barrel. When the turning angular displacement of the rigid body is very small, we can make it similar to

$$
\left\{\begin{array}{l}
l_{0}=l_{2}-l_{1} \\
\ddot{\theta}=\frac{\ddot{s}_{1}-\ddot{s}_{2}}{l_{0}}
\end{array}\right.
$$

In the formula, $s_{1}$ and $s_{2}$ are the lateral displacement of the action sites before and after of the projectile to the inertial coordinate system. Simultaneous equations (2)and(3), it will be 


$$
\left\{\begin{array}{l}
\ddot{s}_{1}=-g+\left(\frac{1}{m}+\frac{l_{1}^{2}}{J}\right) N_{1}+\left(\frac{1}{m}-\frac{l_{1} l_{2}}{J}\right) N_{2}=-g+J_{1} N_{1}+J_{3} N_{2} \\
\ddot{s}_{2}=-g+\left(\frac{1}{m}-\frac{l_{1} l_{2}}{J}\right) N_{1}+\left(\frac{1}{m}+\frac{l_{2}^{2}}{J}\right) N_{2}=-g+J_{3} N_{1}+J_{2} N_{2}
\end{array}\right.
$$

In the formula, $J_{1}=\frac{1}{m}+\frac{l_{1}^{2}}{J}, J_{2}=\frac{1}{m}+\frac{l_{2}^{2}}{J}, J_{3}=\frac{1}{m}-\frac{l_{1} l_{2}}{J}$

\section{Equation Solving}

Assuming that the projectile do uniformly accelerated motion along the barrel, considering the inertia effect, then

$$
\frac{d^{2} y(x, t)}{d t^{2}}=\frac{\partial^{2} y(x, t)}{\partial t^{2}}+2 v \frac{\partial^{2} y(x, t)}{\partial x \partial t}+v^{2} \frac{\partial^{2} y(x, t)}{\partial x^{2}}+a \frac{\partial y(x, t)}{\partial t}
$$

In the formula (5), the first item on the right is the transport acceleration of barrel, the second is the coriolis acceleration caused by the moving load, the third is the centripetal acceleration and the fourth is the normal component of acceleration. The equation (5) can be obtained by $s_{i}=s_{i r}+y(x, t) \delta\left(\xi_{i}-x\right)$, equation (5) can be expressed as:

$$
\ddot{s}_{i}=\ddot{s}_{i r}+\sum_{j=1}^{N} \ddot{q}_{j}(t) \varphi_{j}\left(\xi_{i}\right)+2 v \sum_{j=1}^{N} \dot{q}_{j}(t) \varphi^{\prime}{ }_{j}\left(\xi_{i}\right)+v^{2} \sum_{j=1}^{N} q_{j}(t) \varphi^{\prime \prime}{ }_{j}\left(\xi_{i}\right)+a \sum_{j=1}^{N} q_{j}(t) \varphi^{\prime}{ }_{j}\left(\xi_{i}\right)
$$

In the formula (6), $s_{1 r}$ and $s_{2 r}$ are the lateral displacement of the action sites before and after of the projectile to the axis of barrel. According to the literature [3], the normal impact force $P_{1}$ and $P_{2}$ could be calculated by formula (7).

$$
P_{i}=\left\{\begin{array}{cl}
c_{i} \dot{s}_{i r}+k_{i}\left(\left|s_{i r}\right|-B\right)^{n} \operatorname{sgn}\left(s_{i r}\right) & \left|s_{i r}\right|>B \\
0 & \left|s_{i r}\right| \leq B
\end{array}\right.
$$

According to the literature [1],the vibration equation of barrel could be solved by using the modal expansion method, that was $y(x, t)=\sum_{i=1}^{\infty} q_{i}(t) \varphi_{i}(t)$. In this formula, $\varphi_{i}(t)$ is the $i$ order vibration mode function, and $q_{i}(t)$ is the $i$ order modal coordinates.

$$
\varphi_{i}(t)=\cosh \beta_{i} x-\cos \beta_{i} x-\frac{\sinh \beta_{i} l-\sin \beta_{i} l}{\cosh \beta_{i} l+\cos \beta_{i} l}\left(\sinh \beta_{i} x-\sin \beta_{i} x\right)
$$

In the formula(8), $\beta_{i}=\frac{i-0.5}{l} \pi$. By formula (2) to (8) can introduce:

$$
\left\{\begin{array}{c}
\ddot{q}_{i}(t)+2 H \omega_{i} \dot{q}_{i}(t)+\omega_{i}^{2} \ddot{q}_{i}(t)=\frac{P_{1} \varphi_{i}\left(\xi_{1}\right)+P_{2} \varphi_{i}\left(\xi_{2}\right)}{M_{i}} \\
\ddot{s}_{1 r}+\sum_{j=1}^{N} \ddot{q}_{j}(t) \varphi_{j}\left(\xi_{1}\right)+2 v \sum_{j=1}^{N} \dot{q}_{j}(t) \varphi^{\prime}{ }_{j}\left(\xi_{1}\right)+v^{2} \sum_{j=1}^{N} q_{j}(t) \varphi^{\prime \prime}{ }_{j}\left(\xi_{1}\right)+a \sum_{j=1}^{N} q_{j}(t) \varphi^{\prime}{ }_{j}\left(\xi_{1}\right)=-g+J_{1} N_{1}+J_{3} N_{2} \\
\ddot{s}_{2 r}+\sum_{j=1}^{N} \ddot{q}_{j}(t) \varphi_{j}\left(\xi_{2}\right)+2 v \sum_{j=1}^{N} \dot{q}_{j}(t) \varphi^{\prime}{ }_{j}\left(\xi_{2}\right)+v^{2} \sum_{j=1}^{N} q_{j}(t) \varphi^{\prime \prime}{ }_{j}\left(\xi_{2}\right)+a \sum_{j=1}^{N} q_{j}(t) \varphi^{\prime}{ }_{j}\left(\xi_{2}\right)=-g+J_{3} N_{1}+J_{2} N_{2}
\end{array}\right.
$$

In the formula, $\omega_{i}$ is the $i$ order natural frequency of barrel, $\omega_{i}=\beta^{2}{ }_{i} \sqrt{\frac{E I}{\rho A}} \cdot M_{i}$ is the $i$ order mode mass, $M_{i}=\rho A \int_{0}^{l} \varphi^{2}{ }_{i}(x) d x$. The formula (9) is the coupling vibration equation of projectile and barrel. 


\section{Calculation Examples}

Case (1): Effect of acceleration of projectile on vibration of barrel

In order to verify the accuracy and reliability of the model, we calculated the dynamic response of barrel under the action of projectile. Without consideration of the gap between projectile and barrel . We assumed that the gap between projectile and barrel $B=0$, the length of barrel $l=6.5 m$, the cross-sectional area of barrel $A=0.0075 \mathrm{~m}^{2}$, the distance from the centering department before projectile to the Link Belt $l_{0}=0.3 m$, the distance from the centering department before projectile to the centroid of projectile $l_{1}=0.2 m$, the distance from the Link Belt to the centroid of projectile $l_{2}=0.1 \mathrm{~m}$, the elastic modulus $E=2.1 \times 10^{11} \mathrm{pa}$, the cross-section moment of inertia $I=4.91 \times 10^{-6} \mathrm{~m}^{4}$, the mass of projectile $m=30.73 \mathrm{~kg}$. The projectile do the uniformly accelerated motion with the acceleration of $a=3 \times 10^{4} \mathrm{~m} / \mathrm{s}^{2}, a=4 \times 10^{4} \mathrm{~m} / \mathrm{s}^{2}, a=5 \times 10^{4} \mathrm{~m} / \mathrm{s}^{2}$ and $a=\times 10^{4} \mathrm{~m} / \mathrm{s}^{2}$ in chamber, respectively. The calculated results were shown in figure 2 .

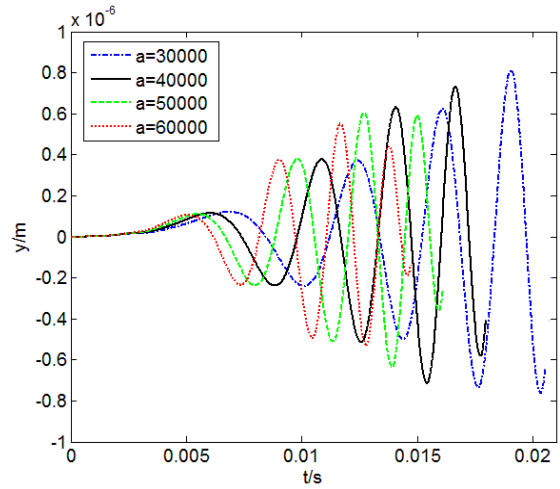

Fig.2 Effect of acceleration of projectile on vibration of barrel

Figure 2 showed that the amplitude of gun muzzle increased with the decreasing of the acceleration of projectile without considering the gap condition. This result was consistent with the literature[1], which verified the accuracy of the model.

Case (2):Effect of gap between projectile and barrel on vibration of barrel

When considering the gap between projectile and barrel, we assumed that $B=0.2 \mathrm{~mm}$, and the projectile do the uniformly accelerated motion with the acceleration of $a=3 \times 10^{4} \mathrm{~m} / \mathrm{s}^{2}$, $a=4 \times 10^{4} \mathrm{~m} / \mathrm{s}^{2}, a=5 \times 10^{4} \mathrm{~m} / \mathrm{s}^{2}$ and $a=6 \times 10^{4} \mathrm{~m} / \mathrm{s}^{2}$ in chamber ,respectively. The calculated results were shown in figure 3 .

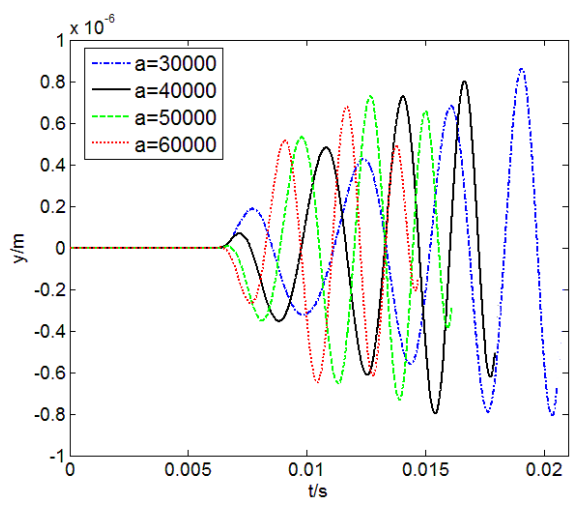

Fig.3 Effect of acceleration of projectile on vibration of barrel under gap condition

Figure 3 showed that the amplitude of barrel increased with the decreasing of the acceleration of projectile with uniformly accelerated motion when considering the gap between projectile and barrel. But the amplitude of barrel was larger than that without considering the gap condition.

In order to further study the influence of the vibration of the gap between projectile and barrel on the barrel, we respectively set the gap $B=0 \mathrm{~mm}, 0.2 \mathrm{~mm}, 0.5 \mathrm{~mm}, 0.8 \mathrm{~mm}$, and the acceleration of projectile $a=5 \times 10^{4} \mathrm{~m} / \mathrm{s}^{2}$. Other parameters are the same as above. The calculated results are shown in figure 4. 


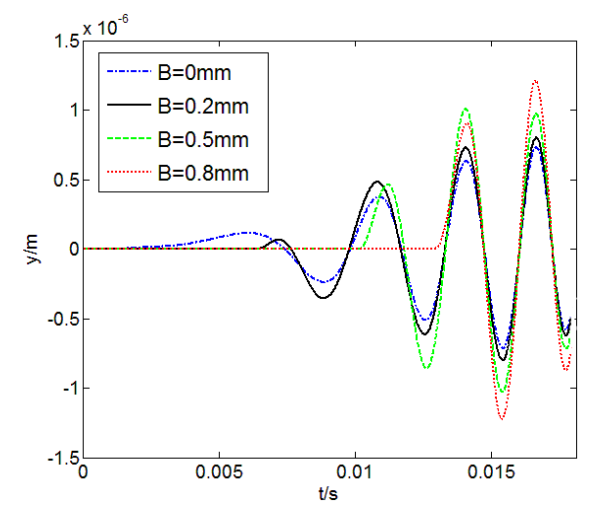

Fig.4 Effect of gap between projectile and barrel on vibration of barrel

Figure 4 showed that the amplitude of barrel increases with the increasing of the gap between projectile and barrel with the same acceleration of projectile. The gap plays an important role in the initial disturbance of projectile. So study the gap between projectile and barrel is very important to the coupling effect of gun.

Case (3): Continuous firing

Next, we further discussed the Lateral vibration characteristics of the barrel excitated by the moving projectile under the condition of continuous firing. Take the projectile which were two consecutive fired as an example, We assumed that the second projectiles entering the chamber with constant acceleration while the first projectiles just fly out of muzzle. Then the barrel excitated by the second projectile had the following initial conditions:

$$
\left.\frac{\partial y(x, t)}{\partial t}\right|_{t=t_{g}}=A,\left.\quad y(x, t)\right|_{t=t_{g}}=B
$$

In the formula, $y(x, t)$ is the deflection of the barrel excitated by the second projectile. $t_{g}$ is the firing interval. $A$ and $B$ are the initial values of the barrel excitated by the second projectile, and the initial values are determined by the values at final conditions of the barrel excitated by the first projectile. we set the gap between projectile and barrel $B=0.4 \mathrm{~mm}$, and the acceleration of projectile $a=4 \times 10^{4} \mathrm{~m} / \mathrm{s}^{2}$. The results were shown in figure 5 .
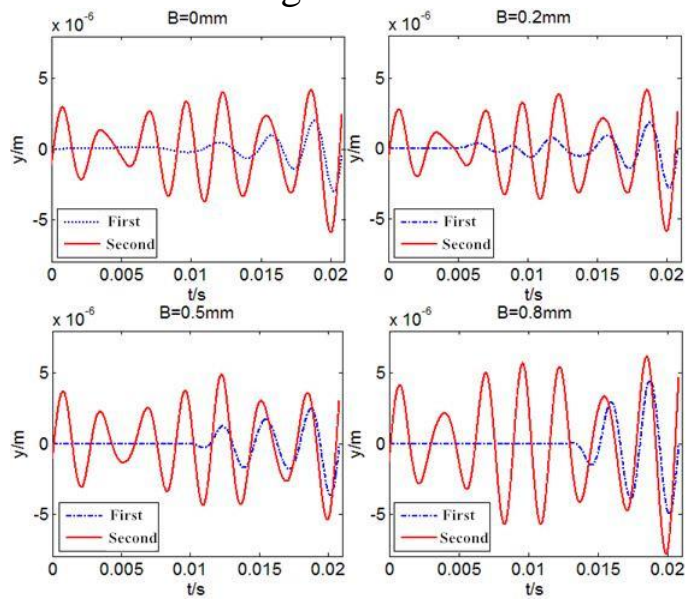

Fig.5 Effect of continuous firing on vibration of barrel

The figure 5 showed that the amplitude of the barrel which was excitated by projectile under the condition of continuous firing was larger than the single projectile. The vibration of barrel was increased because the vibration of barrel under the excitation of the second projectile was affected by the first. That was to say the continuous firing would increase the amplitude of barrel, resulting in decreased accuracy of firing. At the same time, it could be found that the vibration of muzzle was more complex and the vibration frequency of barrel was higher under the condition of continuous firing.

\section{Case (4): Exist eccentric mass}


Due to the action of rifling, the projectile along the direction of axis of barrel in the chamber not only for high-speed translational motion, but also for high-speed rotary motion. If the eccentric mass of projectile exists at this time, the high speed rotation of projectile will produce a great centrifugal force, which will affect the force between projectile and barrel. Considering the effect of centrifugal force, the differential equationthe of movement of projectile is:

$$
m\left(\frac{d^{2} s_{r}(t)}{d t^{2}}+\frac{d^{2} y(x, t)}{d t^{2}} \delta\left(\xi_{0}-x\right)\right)=\left(P_{1}+P_{2}\right)-\left(m g+m R \varpi^{2} \sin (\varpi t)\right)
$$

In the formula, $R$ is the eccentricity of projectile, $\varpi$ is the speed of projectile in the chamber. For the uniform twist rifling, $\varpi=\frac{2 \pi}{\eta d} v, \eta$ is the twist rate, and $d$ is the inner diameter of barrel.

In the following, we studyed the effects of eccentric mass of projectile on the vibration of barrel under different gaps between projectile and barrel. We set $R=10^{-3} d, \eta=27$, and the other parameters were kept constant. The calculation results were shown in Figure 6.
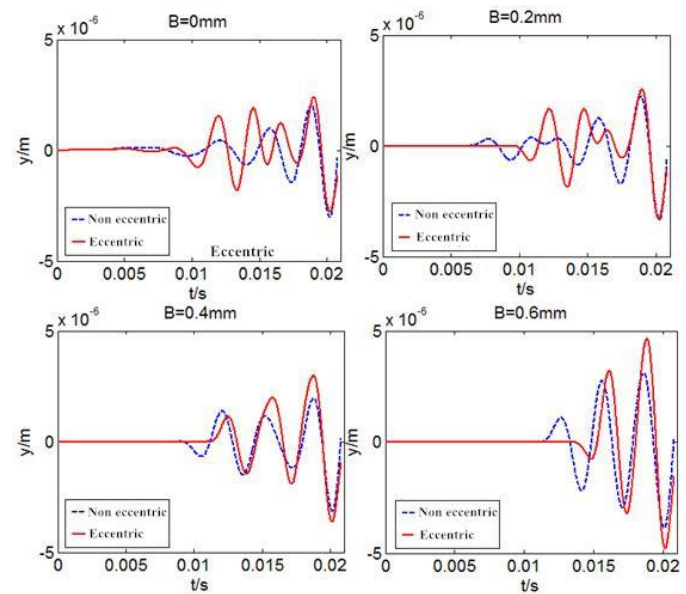

Fig.6 Effect of eccentric mass on vibration of barrel

From Figure 6, we could see that when the gap between projectile and barrel become smaller, the vibration frequency of barrel under the function of eccentric mass was higher than without it, but the changes of amplitude of barrel was not obvious. As the the gap between projectile and barrel increased, the vibration frequency of barrel under the function of eccentric mass was getting closer to that without it, and the amplitude of barrel was higher than without it, too.

\section{Conclusion}

The paper studied the vibration characteristics of barrel. Considering the effect of Inertia and the contact condition between projectile and barrel, we established the vibration equation of barrel excited by the accelerated projectile, and used the modal analysis method to solve this equation. The results showed that :

Considering the condition of the gap between projectile and barrel, the amplitude of barrel increased with the decreasing of acceleration of projectile with uniformly accelerated motion, and the amplitude of barrel increased with the increasing of the gap with the same acceleration of projectile. So the gap played an important role in the initial disturbance of projectile.

The amplitude of the barrel which was excitated by projectile under the condition of continuous firing was larger than the single projectile. The vibration of barrel was increased because the vibration of barrel under the excitation of the second projectile is affected by the first. That is to say the firing ripples will increase the amplitude of barrel, resulting in decreased accuracy of firing.

When the gap between projectile and barrel becomes smaller, the vibration frequency of barrel under the function of eccentric mass was higher than without it, but the changes of amplitude of barrel was not obvious. As the gap between projectile and barrel increased, the vibration frequency 
of barrel under the function of eccentric mass was getting closer to that without it, and the amplitude of barrel was higher than without it, too.

The above work was mainly about the derivation and analysis of the theoretical formula, and the calculation of examples. All of these give the effect of gap between projectile and barrel, continuous firing and the eccentric mass of projectile on the vibration of barrel.

The results showed that the reasonable control of the acceleration of projectile in chamber, the reduction of the gap between projectile and barrel and the eccentric mass of projectile were conducive to reducing the vibration of muzzle caused by artillery launch. The research results can provide reference for the overall design of gun.

\section{References}

[1] Deshi Wang:Theory of Gun vibration (Weapons industry press, Beijing 2015, In Chinese)

[2] Deshi Wang and Yuedong Shi : Journal of Dynamics and Control, 10(04) (2012), p. 304-323. (In Chinese)

[3] Ning Liu and Guolai Yang: Journal of Ballistics, 02(2010), p. 67-70. (In Chinese)

[4] Mu Jiang and Xifu Guo: Journal of Ballistics, 03(2002), p. 57-62. (In Chinese)

[5] Mu Jiang : Mechanics and Engineering, 06(2002), p. 44-47. (In Chinese)

[6] Mu Jiang : Journal of Ballistics, 01(2002), p. 77-83. (In Chinese) 